\title{
Full counting statistics of Luttinger liquid conductor
}

\author{
D. B. Gutman ${ }^{1}$, Yuval Gefen ${ }^{2}$, and A. D. Mirlin ${ }^{3,4,5}$ \\ ${ }^{1}$ Department of Physics, Bar Ilan University, Ramat Gan 52900, Israel \\ ${ }^{2}$ Dept. of Condensed Matter Physics, Weizmann Institute of Science, Rehovot 76100, Israel \\ ${ }^{3}$ Institut für Nanotechnologie, Karlsruhe Institute of Technology, 76021 Karlsruhe, Germany \\ ${ }^{4}$ Institut für Theorie der kondensierten Materie, Karlsruhe Institute of Technology, 76128 Karlsruhe, Germany \\ ${ }^{5}$ Petersburg Nuclear Physics Institute, 188300 St. Petersburg, Russia
}

(Dated: September 13, 2018)

\begin{abstract}
Non-equilibrium bosonization technique is used to study current fluctuations of interacting electrons in a single-channel quantum wire representing a Luttinger liquid (LL) conductor. An exact expression for the full counting statistics of the transmitted charge is derived. It is given by Fredholm determinant of the counting operator with a time dependent scattering phase. The result has a form of counting statistics of non-interacting particles with fractional charges, induced by scattering off the boundaries between the LL wire and the non-interacting leads.
\end{abstract}

PACS numbers: 73.23.-b, 73.40.Gk, 73.50.Td

Fluctuations are among the most fundamental concepts arising in statistical physics. In recent years, nonequilibrium noise was measured in a variety of electronic systems, such as quantum point contacts[1], diffusive mesoscopic conductors 2], and fractional quantum Hall edges [3]; see Ref. 4 for review. With the decrease of a sample size, characterization of current fluctuations by the second moment only becomes insufficient. This has triggered recent interest in higher-order correlation functions of current statistics. The third cumulant of the noise was measured in recent experiments [5]. A more complete characterization of current fluctuations is the full counting statistics (FCS), introduced by Levitov et al. [6]. In its full extent this fascinating theoretical approach yields information about all moments of the number of electrons transferred (over a given time interval) through a given terminal in a multi-terminal system, current cross-correlations and entanglement, and large current fluctuations.

For non-interacting systems, the FCS of nonequilibrium fluctuations is well understood by now within several complementary approaches, including the Fredholm determinant formalism [6, 7], the $\sigma$-model fieldtheoretical description [8], as well as the kinetic theory of fluctuations and related methods [9].

Much less remains known concerning fluctuations in interacting systems. This problem is of particular interest at low dimensions, where interaction affects the nature of the system at hand in an essential way. This is, in particular, the case for FCS of current through a quantum dot in the Coulomb blockade regime [10]. The interaction also affects dramatically the physics of onedimensional (1D) fermionic systems where a strongly correlated state - Luttinger liquid (LL) - is formed[11]. Experimental realizations of LL include carbon nanotubes, semiconductor, metallic and polymer nanowires, as well as quantum Hall edges. Recent experiments studied the shot noise in carbon nanotubes [12]. Non-equilibrium physics of carbon nanotubes and quantum Hall edges has been explored through tunneling spectroscopy [13] and Mach-Zehnder-iterferometry [14] respectively. The LL description is also relevant to interacting $1 \mathrm{D}$ bosonic systems, cf. e.g. recent experiments on ultracold atomic gases that probe statistical properties of inteference contrast, thus obtaining information on the full distribution of quantum noise [15].

Previous theoretical work on current fluctuations in LL mainly focussed on the second moment (shot noise) [16]. The most intriguing observation was related to manifestations of fractional charges in shot noise. However, full understanding of the nature of charge transfer processes (and, in particular, of charge fractionalization) requires the analysis of the FCS. The latter has been studied in a biased LL with an impurity 17]. While an analytical solution via the thermodynamic Bethe ansatz can be found [17], it is in general implicit and very cumbersome.

In the present work we study the statistical properties of current fluctuations in non-equilibrium LL conductor with arbitrary energy distribution of right- and left-movers. Employing the recently developed nonequilibrium bosonization technique [18], we find an exact solution of this problem. We show that the full distribution of the current noise is closely related to the phenomenon of charge fractionalization. Specifically, we demonstrate that the FCS of LL reduces to the one of a non-interacting model with fractional charges calculated below.

We consider a LL conductor geometry schematically shown in Fig. 1 Our goal is to solve FCS problem in the presence of electron interaction. We assume that the spatially dependent interaction strength $g(x)$ vanishes outside the interval $[-L / 2, L / 2]$; this way of modeling leads was introduced in Refs. 19 21 to study the conductance of LL wire. To describe the FCS of charge transfer, we define a probability distribution function $p_{\tau}(n)$ for $n$ electrons to pass through the cross-section during the time 


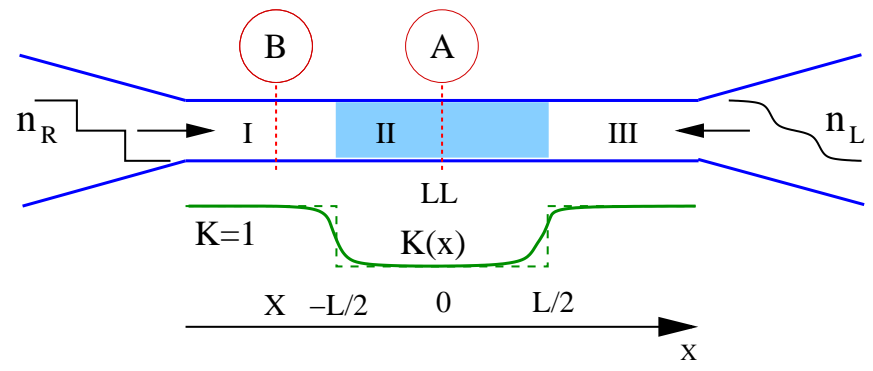

FIG. 1: Schematic view of noise detection in LL conductor. The positions of the charge detector inside (A) and outside (B) of interacting region are indicated. The $x$-dependent LL parameter $K(x)$ for smooth (solid line) and sharp (dashed line) boundaries is also shown.

interval $\tau$. It is convenient to introduce a generating fuction $\kappa(\lambda)=\sum_{n=-\infty}^{\infty} p_{\tau}(n) e^{i n \lambda}$.

Let us first discuss the non-interacting case. In that case $\kappa(\lambda)$ has been calculated in Ref. 6 by means of Landauer scattering-state approach. For an ideal quantum wire (with no scattering inside the wire) the generating function of FCS is given by

$$
\kappa(\lambda)=\Delta_{R}\left[\delta_{R}(t)\right] \Delta_{L}\left[\delta_{L}(t)\right] .
$$

Here $\eta=R, L$ labels right-and left-movers and

$$
\Delta_{\eta}\left[\delta_{\eta}(t)\right]=\operatorname{det}\left[1+\left(e^{-i \delta_{\eta}}-1\right) n_{\eta}\right]
$$

is a Fredholm determinant (of Toeplitz type) of the counting operator built of fermionic distribution function $n_{\eta}(\epsilon)$ and a time-dependent scattering phase $\delta_{\eta}(t)$, with $\epsilon$ and $t$ understood as canonically conjugate variables. In Eq. (11) and below we assume normalization of the determinant $\Delta_{\eta}$ to its value for equilibrium, zero-temperature distribution. The phase $\delta_{\eta}(t)$ is given by

$$
\delta_{\eta}(t)=\lambda \eta w_{\tau}(t, 0),
$$

where we have defined a window function $w_{\tau}(t, \tilde{t})=$ $\theta(\tilde{t}-t)-\theta(\tilde{t}-t-\tau)$. We use the convention that in formulas $\eta$ is understood as $\eta= \pm 1$ for right-/left-movers. In the long time limit the Fredholm determinant (2) can be easily evaluated analytically; in a more general situation it can be efficiently studied numerically [22]. Below we show that for the interacting case, the generating function of the FCS obeys the form of Eq. (11). All electron interaction effects are encoded in the time dependent scattering phases $\delta_{\eta}(t)$.

On the microscopic level the problem is described by the Keldysh action $S=S_{0}[\psi]+S_{\text {ee }}[\psi]$, written in terms of fermionic fields 24],

$$
\begin{aligned}
& S_{0}[\psi]=i \sum_{\eta} \int_{c} d t \int d x \psi_{\eta}^{\dagger} \partial_{\eta} \psi_{\eta}, \\
& S_{\mathrm{ee}}[\psi]=-\sum_{\eta} \int_{c} d t \int d x g(x)\left(\rho_{\eta} \rho_{-\eta}+\rho_{\eta} \rho_{\eta}\right) .
\end{aligned}
$$

Here $\rho_{\eta}=\psi_{\eta}^{\dagger} \psi_{\eta}$ are density fields, $\partial_{\eta}=\partial_{t}+\eta v \partial_{x}$, $v$ is the Fermi velocity. The non-equilibrium bosonization approach allows us to reformulate this theory in terms of bosonic (density) fields. The interacting part of the action, $S_{e e}$, is already expressed in terms of density modes $\rho_{\eta}$. Following rotation in Keldysh space $S_{e e}=\sum_{\eta \eta^{\prime}} \int d t d x g(x) \rho_{\eta} \bar{\rho}_{\eta^{\prime}}$, while the free part of the action, where information concerning the state of the non-interacting fermionic system is encoded, reads

$$
S_{0}=\sum_{\eta}\left(-\rho_{\eta} \Pi_{\eta}^{a^{-1}} \bar{\rho}_{\eta}-i \ln Z_{\eta}\left[\bar{\chi}_{\eta}\right]\right) .
$$

Here we have decomposed the bosonic variables into classical and quantum components, $\rho, \bar{\rho}=\left(\rho_{+} \pm \rho_{-}\right) / \sqrt{2}$, where the indices + and - refer to the two branches of the Keldysh contour; $\Pi_{\eta}^{a}$ is the advanced component of polarization operator, and $Z_{\eta}\left[\overline{\chi_{\eta}}\right]$ is a partition function of free fermions moving in the field

$$
\bar{\chi}_{\eta}=\Pi_{\eta}^{a^{-1}} \bar{\rho}_{\eta}, \quad \Pi_{\eta}^{a}(\omega, q)=\eta q / 2 \pi(\eta v q-\omega-i 0) .
$$

Expansion of $Z_{\eta}\left[\bar{\chi}_{\eta}\right]$ in $\bar{\chi}_{\eta}(t, x)$ generates an infinite series, $i \ln Z_{\eta}\left[\bar{\chi}_{\eta}\right]=\sum_{n}(-1)^{n+1} \bar{\chi}_{\eta}^{n} \mathcal{S}_{\eta n} / n$, governed by irreducible fermionic density correlation functions, $\mathcal{S}_{\eta n}\left(t_{1}, x_{1} ; \ldots ; t_{n}, x_{n}\right) \equiv\left\langle\left\langle\rho_{\eta 1} \rho_{2} \ldots \rho_{\eta n}\right\rangle\right\rangle$, representing cumulants of quantum noise [6].

To find the generating function for the FCS of charge transferred through a cross-section $x=X$ during a time interval $\tau$, one needs to calculate the correlation function

$$
\kappa(\lambda, X)=\left\langle e^{i \lambda Q(\tau, X)} e^{-i \lambda Q(0, X)}\right\rangle,
$$

where $Q(t, X)=\int_{-\infty}^{X}\left(\rho_{R}(x)+\rho_{L}(x)\right) d x$ is the electric charge situated on the left side of the point $X$ at the time $t$. We note a mathematical similarity between the problem of FCS and that of tunneling spectroscopy [18]. The latter amounts to evaluation of a single-particle Green function. In both cases one needs to find a correlation function of exponentials of bosonic operators that are linear combinations of the right and left density fields. A major difference stems from the fact that the operators $e^{ \pm i \lambda Q}$ in Eq. (6) contain a sum of left and right densities in the exponential, whereas the fermionic operators $\psi_{\eta}$, $\psi_{\eta}^{\dagger}$ contain only $\rho_{\eta}$.

We now proceed with calculation of the generating function (6); technical details are outlined in the Supplementary Material [23]. We find that the FCS generating function has the form of Eq. (1), as in the case of free fermions. The time dependent scattering phases $\delta_{\eta}(t)$ are expressed through plasmon reflection and transmission coefficients $r_{\eta}, t_{\eta}$ at the left (I/II; $\left.\eta=L\right)$ and right (II/III; $\eta=R$ ) boundaries; $r_{\eta}^{2}+t_{\eta}^{2}=1$. In general, multiple scattering off these boundaries will give rise to an infinite sequence of scattering phase pulses [cf. Eq. (7)]. If the boundary is smooth (on the scale of the plasmon wave length), there is no plasmon reflection, $r_{\eta} \simeq 0$. In the opposite limit of a sharp boundary we have $r_{\eta}=(1-K) /(1+K)$. 

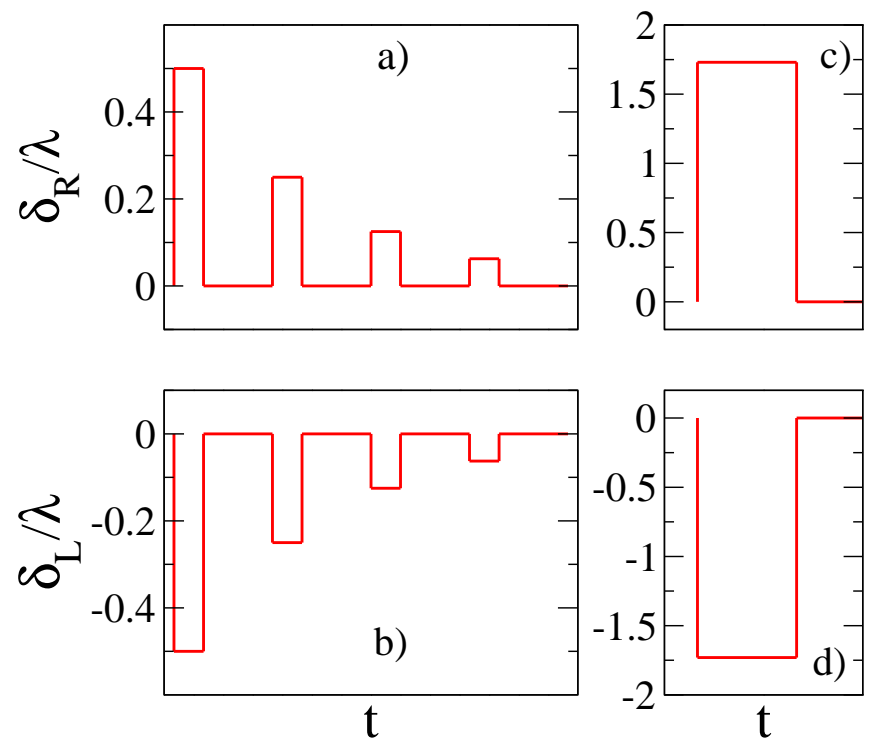

FIG. 2: Phases $\delta_{\eta}$ entering Eq. (1) for the measuring device located at $X=0$, for sharp (a,b) and adiabatic (c,d) boundaries $(K=1 / 3)$.

In contrast with the free fermion case, the statistics of current fluctuations in the LL conductor depend on the position of the measuring device (see Fig.1). Let us first consider the case where the current fluctuations are measured in the middle of an interacting region $(X=0$, position A in Fig [1). We thus find [23] that time dependent scattering phases consist of a sequence of pulses

$$
\delta_{\eta}(t)=\sum_{n=0}^{\infty} \delta_{\eta, n} w_{\tau}\left(t, t_{n}\right)
$$

with partial phase shifts

$$
\begin{aligned}
& \delta_{\eta, 2 n}=\eta \lambda t_{-\eta} \sqrt{K} r_{\eta}^{n} r_{-\eta}^{n} \equiv \eta \lambda e_{\eta, 2 n}^{*} \\
& \delta_{\eta, 2 n+1}=\eta \lambda t_{-\eta} \sqrt{K} r_{\eta}^{n+1} r_{-\eta}^{n} \equiv \eta \lambda e_{\eta, 2 n+1}^{*} .
\end{aligned}
$$

The beginning of the $n$-th pulse occurs at time $t_{n}=$ $(n+1 / 2-1 / 2 K) L / u$. The sequence of pulses $\delta_{\eta}(t)$ is shown in Fig. 2 for the limiting cases of sharp and smooth boundaries between the interacting and non-interacting regions.

In the limit of very low frequencies $(\tau \gg L / u)$ all pulses (7) overlap and the scattering phase can be approximated by $\delta_{\eta}(t) \simeq w_{\tau}(t, 0) \sum_{n=0}^{\infty} \delta_{\eta, n}$. In this limit of long wavelength the interacting/non-interacting boundaries appear to be sharp. This yields $\sum_{n=0}^{\infty} \delta_{\eta, n}=\eta \lambda$. As expected [19 21] the effects of interaction in this limit disappear and one is back to the FCS of free fermions [6].

In the opposite case $(\tau \ll L / u)$ the interference between different plasmon pulses may be neglected and the result splits into a product

$$
\Delta_{\eta}\left[\delta_{\eta}(t)\right] \simeq \prod_{n=0}^{\infty} \Delta_{\eta \tau}\left(\delta_{\eta, n}\right), \quad \delta_{\eta, n} \equiv \eta \lambda e_{\eta, n}^{*},
$$

with each factor representing a contribution of a single phase pulse $\delta_{\eta, n}(t)=\delta_{\eta, n} w_{\tau}(t, 0)$. Remarkably, plasmon scattering gives rise to charge fractionalization, which splits the scattering phase into an infinite series of pulses. On a technical level, the Fredholm determinant of the counting operator is now replaced by an infinite product of determinants, each calculated with a corresponding scattering phase. The FCS of the LL then becomes a superposition of FCS of non-interacting electrons with fractional charges $e_{\eta, n}^{*}$. For the case of smooth boundaries we get only one fractional charge, $e_{\eta, 0}^{*}=\sqrt{K}$. In the opposite limit of sharp boundaries, we obtain the sequence of fractional charges of the form $e_{\eta, n}^{*}=2 K(1-K)^{n} /(1+K)^{n+1}$.

Focussing on the regime where the counting interval $\tau$ is long compared to the inverse energy scale of the distributions $n_{\eta}(\epsilon)$, we express $\Delta_{\eta \tau}(\delta)$ in the form

$$
\ln \Delta_{\eta \tau}(\delta)=\tau \int \frac{d \epsilon}{2 \pi}\left\{\ln \left[1+\left(e^{-i \delta}-1\right) n_{\eta}(\epsilon)\right]+i \delta \theta(-\epsilon)\right\} .
$$

Substituting (11) in Eqs. (10), (10) and expanding in $\delta$, one can find explicitly all moments of noise.

Consider now the FCS outside the interacting region, e.g. at $X<-L / 2$ (B in Fig (1). The analysis follows closely the one outlined above, hence we present only the final result. The right and left scattering phases are

$$
\begin{aligned}
\delta_{R}(t) & =\lambda\left[w_{\tau}\left(t, \frac{X}{v}\right)-r_{L} w_{\tau}\left(t, \frac{L+X}{v}\right)\right. \\
& \left.+r_{R} t_{L}^{2} \sum_{n=0}^{\infty} r_{L}^{n} r_{R}^{n} w_{\tau}\left(t, \frac{L+X}{v}+\frac{2(n+1) L}{u}\right)\right], \\
\delta_{L}(t) & =-\lambda t_{L} t_{R} \sum_{n=0}^{\infty} r_{L}^{n} r_{R}^{n} w_{\tau}\left(t, \frac{2 n L}{u}-\frac{X}{v}+\frac{v-u}{v u} L\right) .
\end{aligned}
$$

Current fluctuations measured in the non-interacting region at finite frequency $(\tau \ll L / u)$ differ from those at the interacting part of LL (8). Here too the FCS takes the form of superposition of non-interacting FCS's, but with a new set of fractional charges:

$$
\begin{aligned}
& e_{R, 0}^{*}=1, \quad e_{R, 1}^{*}=-r_{L}, \quad e_{R, n+2}^{*}=t_{L}^{2} r_{L}^{n} r_{R}^{n+1}, \\
& e_{L, n}^{*}=t_{L} t_{R} r_{L}^{n} r_{R}^{n} \quad(n=0,1, \ldots) .
\end{aligned}
$$

In the universal ultra-low-frequency limit $(\tau \gg L / u)$ the effects of interaction again disappear, and the result for the FCS of free fermions is recovered.

To conclude we provide a brief overview of the physics discussed here. We have calculated the FCS in a LL conductor. The result is cast in terms of a Fredholm determinant of the counting operator. The boundaries between the interacting and non-interacting regions of the wire give rise to (in general, multiple) plasmon scattering. This generates plasmon wavelets corresponding to fractionally charged "particles". As a result, for a counting 
time interval $\tau$ small compared with the plasmon timeof-flight through the interacting region, $L / u$, the FCS is a superposition of counting statistics of non-interacting particles with fractional charges $e_{\eta, n}^{*}$. Let us stress that the FCS contains more complete information about the system than the second moment (noise). As an example, for a symmetric system $\left(r_{L}=r_{R}\right)$ the noise acquires the factor $K$ due to interaction. By itself, this is not sufficient to make a conclusion about the value of fractional charges and the character of correlations between them.

The fractionalization process that manifests itself in the FCS caculated above is solely due to the interacting/non-interacting boundary scattering [20]. Fractionalization due to tunneling into a LL 25] does not show up here: technically this is since the operator $Q$ in Eq. (6) is determined by a sum of $\rho_{L}$ and $\rho_{R}$.

How is the notion of charge fractionalization compatible with charge quantization? The above analysis was based on the bosonization approach with the density $\rho_{\eta}$ slowly varying on the scale of the inverse Fermi wave vector $k_{F}^{-1}$. This corresponds to the situation where the measurement procedure is smooth on the scale $k_{F}^{-1}$. In this situation the above results are valid for any counting field $\lambda$ : our detector may count a fractional charge residing in some volume of space. In the opposite limit, where the spatial resolution of our measurement is sharp on the scale $k_{F}^{-1}$, the measured charge should be integer, i.e. the FCS should satisfy $\kappa(\lambda)=\kappa(\lambda+2 \pi)$. In the bosonization formalism, charge quantization emerges by taking into account fast oscillatory contributions to the density $\rho_{\eta}$ [26]. This will not affect the moments of FCS (which are obtained as derivatives of $\kappa(\lambda)$ at $\lambda=0$ ) and, more generally, the form of $\kappa(\lambda)$ in the range $[-\pi, \pi]$. Beyond this interval, the FCS will be continued periodically, in agreement with the charge quantization requirement.

This work was supported by GIF, DFG Center for Functional Nanostructures, Einstein Minerva Center, US-Israel BSF, ISF, Minerva Foundation, DFG SPP 1285, EU GEOMDISS, and Rosnauka 02.740.11.5072.

[1] M. Reznikov et al., Phys. Rev. Lett. 75, 3340 (1995); A. Kumar et al., Phys. Rev. Lett. 76, 2778 (1996).

[2] A.H. Steinbach, J.M. Martinis, and M.H. Devoret, Phys. Rev. Lett. 76, 3806 (1996); R.J. Schoelkopf et al., Phys. Rev. Lett 78, 3370 (1997).

[3] R. de Picciotto et al., Nature 389, 162 (1997); L. Saminadayar et al., Phys. Rev. Lett. 79, 2526 (1997); M. Reznikov et al., Nature 399, 238 (1999); M. Reznikov et al., Phys. Rev. Lett. 75, 3340 (1995).

[4] Ya.M. Blanter and M. Büttiker, Phys. Rep. 336, 1 (2000).

[5] B. Reulet, J. Senzier, D.E. Prober, Phys. Rev. Lett. 91, 196601 (2003); Yu. Bomze et al., Phys. Rev. Lett. 95, 176601 (2005); G. Gershon et al., Phys. Rev. Lett. 101, 016803 (2008).

[6] L.S. Levitov and G.B. Lesovik, JETP Lett. 58, 230
(1993); L.S. Levitov, H. Lee, and G.B. Lesovik, J. of Math. Phys. 37, 4845 (1996); D.A. Ivanov, H. Lee, and L.S. Levitov, Phys. Rev. B 56, 6839 (1997).

[7] B.A. Muzykantskii and Y. Adamov, Phys. Rev. B 68, 155304 (2003); A. Shelankov and J. Rammer, Europhysics Letters 63, 485 (2003); K. Schoenhammer, Phys. Rev. B 75, 205329 (2007); J.E. Avron et al., Commun. Math. Phys. 280, 807 (2008); F. Hassler et al., Phys. Rev. B 78, 165330 (2008).

[8] Yu. Nazarov, Ann. Phys. (Leipzig) 8, 193 (1999); D.B. Gutman, Y. Gefen, and A.D. Mirlin, in Quantum Noise in Mesoscopic Physics, edited by Yu. Nazarov (Kluwer, Dordrecht, 2003), p. 497.

[9] K.E. Nagaev, Phys. Rev. B 66, 075334 (2002); S. Pilgram et al., Phys. Rev. Lett. 90, 206801 (2003); T. Bodineau and B. Derrida, Phys. Rev. Lett. 92, 180601 (2004); D.B. Gutman, A.D. Mirlin, and Y. Gefen, Phys. Rev. B 71, 085118 (2005).

[10] A.V. Andreev and E.G. Mishchenko, Phys. Rev. B 64, 233316 (2001); D.A. Bagrets and Yu.V. Nazarov, Phys. Rev. B 67, 085316 (2003).

[11] T. Giamarchi, Quantum Physics in One Dimension, (Claverdon Press Oxford, 2004); V.V. Deshpande et al., Nature 464, 209 (2010).

[12] N.Y. Kim et al., Phys. Rev. Lett. 99, 036802 (2007); F. Wu et al., Phys. Rev. Lett. 99, 156803 (2007).

[13] Y.-F. Chen et al., Phys. Rev. Lett. 102, 036804 (2009); C. Altimiras et al., Nature Physics 6, 34 (2010).

[14] Y. Ji et al., Nature 422, 415 (2003); I. Neder et al., Phys. Rev. Lett. 96, 016804 (2006); I. Neder et al., Nature Physics 3, 534 (2007); E. Bieri et al., Phys. Rev. B 79, 245324 (2009).

[15] S. Hofferberth et al., Nature Physics 4, 489 (2008).

[16] C.L. Kane and M.P.A. Fisher, Phys. Rev. Lett. 72, 724 (1994); P. Fendley and H. Saleur, Phys. Rev. B 54, 10845 (1996); V.V. Ponomarenko, Phys. Rev. B 54, 10328 (1996); B. Trauzettel et al., Phys. Rev. Lett. 92, 226405 (2004); A.V. Lebedev, A. Crépieux, and T. Martin, Phys. Rev. B 71, 075416 (2005); I. Safi, C. Bena, and A. Crépieux, Phys. Rev. B 78, 205422 (2008); E. Berg et al., Phys. Rev. Lett. 102, 236402 (2009).

[17] H. Saleur and U. Weiss, Phys. Rev. B 63, 201302 (2001); A. Komnik, B. Trauzettel, and U. Weiss, Annalen der Physik 16, 661 (2007).

[18] D.B. Gutman, Y. Gefen and A.D. Mirlin, arXiv:0906.4076 Phys. Rev. B 81, 085436 (2010).

[19] D.L. Maslov and M. Stone, Phys. Rev. B 52, R5539 (1995).

[20] I. Safi and H.J. Schulz, Phys. Rev. B 52, R17040 (1995).

[21] V.V. Ponomarenko, Phys. Rev. B 52, R8666 (1995).

[22] I. Snyman and Y.V. Nazarov, Phys. Rev. Lett. 99, 096802 (2007); I. Neder and F. Marquardt, New Journal of Physics 9, 112 (2007).

[23] see Supplementary Material

[24] for review of the Keldysh technique see, e.g., J. Rammer and H. Smith, Rev. Mod. Phys. 58, 323 (1986); A. Kamenev and A. Levchenko, Adv. Phys. 58, 197 (2009).

[25] I. Safi, Ann. Phys. 22, 463 (1997); K.-V. Pham, M. Gabay, and P. Lederer, Phys. Rev. B 61, 16397 (2000); K. Le Hur, Phys. Rev. Lett. 95, 076801 (2005); Phys. Rev. B 74, 165104 (2006).

[26] F.D.M. Haldane, J. Phys.C 14, 2585 (1981). 


\section{ONLINE SUPPLEMENTARY MATERIAL}

Here we present details of the calculation of the generating function (6). Despite the fact that the bosonic action (4) contains terms of all orders, evaluation of the generating function can be performed exactly. The point is that the action (4) is linear with respect to the classical component $\rho_{\eta}$ of the density field. Thus, integration with respect to $\rho_{\eta}$ can easily be performed. It gives rise to a $\delta$-function condition for the quantum component $\bar{\rho}_{\eta}$ of the density,

$$
\begin{aligned}
& \partial_{t} \bar{\rho}_{R}+\partial_{x}\left[\left(v+\frac{g}{2 \pi}\right) \bar{\rho}_{R}+\frac{g}{2 \pi} \bar{\rho}_{L}\right]=\frac{\lambda}{2 \pi} j \\
& \partial_{t} \bar{\rho}_{L}-\partial_{x}\left[\left(v+\frac{g}{2 \pi}\right) \bar{\rho}_{L}+\frac{g}{2 \pi} \bar{\rho}_{R}\right]=-\frac{\lambda}{2 \pi} j .
\end{aligned}
$$

Here $j_{\tau}(x, t)$ is a source term,

$$
j_{\tau}(x, t)=\delta(x-X)[\delta(t-\tau)-\delta(t)] / \sqrt{2} .
$$

It remains to solve Eqs. (S1) for $\bar{\rho}_{\eta}$, to calculate $\bar{\chi}_{\eta}$ according to Eq. (5) and then to substitute it into the second term of the bosonic action (41). This yields

$$
Z_{\eta}\left[\bar{\chi}_{\eta}\right]=\Delta_{\eta}\left[\delta_{\eta}(t)\right]
$$

We thus find that the generating function of the FCS problem has the form of Eq. (1), as in the case of free fermions. The time dependent scattering phases $\delta_{\eta}(t)$ are given by the integral of $\bar{\chi}_{\eta}$ along a free-electron trajectory,

$$
\begin{aligned}
\delta_{\eta}(t) & =\sqrt{2} \int_{-\infty}^{\infty} d \tilde{t} \bar{\chi}_{\eta}(\tilde{t}-t, \eta v \tilde{t}) \\
& =-2 \pi \sqrt{2} \eta \lim _{\tilde{t} \rightarrow-\infty} \int_{0}^{\eta v(\tilde{t}+t)} d \tilde{x} \bar{\rho}_{\eta}(\tilde{x}, \tilde{t}) .
\end{aligned}
$$

The second line of Eq. (S4) expresses $\delta_{\eta}(t)$ through the asymptotic behavior of $\bar{\rho}_{\eta}(x, t)$ in the non-interacting parts of the wire (regions I and III in Fig 1).
To find the scattering phases, we now solve the set of linear differential equations (S1). These equations can be conveniently combined into a single differential equation for the current operator $\bar{J}=v\left(\bar{\rho}_{R}-\bar{\rho}_{L}\right)$

$$
\left(\omega^{2}+\partial_{x} u^{2}(x) \partial_{x}\right) \bar{J}(\omega, x)=0, x \neq X
$$

where $u(x)=v / K(x)$ is the plasmon velocity and $K(x)=[1+g(x) / \pi v]^{-1 / 2}$ is the LL parameter. The latter is equal to 1 in the leads and takes a constant value $K$ in the interacting region; the crossover between these values depends on the profile of the interaction. Inside the interacting region $\bar{J}(\omega, x)$ is given by a superposition of the right and left moving plasmon waves:

$$
\bar{J}(\omega, x)=u^{-1 / 2}\left(a e^{i \kappa x}+b e^{-i \kappa x}\right), \quad \kappa=\omega / u .
$$

In the non-interacting regions, an analogous form holds, with the replacement $u \rightarrow v$. The change of interaction strength constitutes a source of plasmon scattering and can be described by plasmons scattering matrices,

$$
S_{L}=\left(\begin{array}{cc}
t_{L} & -r_{L} \\
r_{L} & t_{L}
\end{array}\right), \quad S_{R}=\left(\begin{array}{cc}
t_{R} & r_{R} \\
-r_{R} & t_{R}
\end{array}\right)
$$

The matrices $S_{L, R}$ relate the amplitudes $a, b$ at the left (I/II) and right (II/III) boundaries, respectively; $r_{\eta}^{2}+t_{\eta}^{2}=1$. In general, multiple scattering off these boundaries will give rise to an infinite sequence of scattering phase pulses (cf. Eq. 7). If the boundary is smooth (on the scale of the plasmon wave length), there is no plasmon reflection, $r_{\eta} \simeq 0$. In the opposite limit of a sharp boundary we have $r_{\eta}=(1-K) /(1+K)$.

Using Eqs. (S4), (S5), (S6), and (S7), we obtain the phases $\delta_{\eta}(t)$ as given in the main text of the paper. 